\title{
NF-кB-dependent anti-inflammatory activity of urolithins, gut microbiota ellagic acid-derived metabolites, in human colonic fibroblasts
}

\author{
Antonio González-Sarrías ${ }^{1}$, Mar Larrosa ${ }^{1}$, Francisco Abraham Tomás-Barberán ${ }^{1}$, Piero Dolara ${ }^{2}$ \\ and Juan Carlos Espín ${ }^{1}$ \\ ${ }^{1}$ Department of Food Science and Technology, Research Group on Quality, Safety and Bioactivity of Plant Foods, CEBAS-CSIC, \\ 30100 Campus de Espinardo, Murcia, Spain \\ ${ }^{2}$ Department of Pharmacology, University of Florence, 50139 Florence, Italy
}

(Received 18 August 2009 - Revised 11 February 2010 - Accepted 12 February 2010 - First published online 26 March 2010)

Previous studies have reported the anti-inflammatory properties of pomegranate extracts, suggesting that ellagitannins (ET) and ellagic acid (EA) are the main anti-inflammatory compounds. However, both ET and EA are metabolised in vivo by the gut microbiota to yield urolithins (Uro) which can be found in the gut and in systemic bloodstream. The present study was carried out to evaluate the individual effect of EA and their microbiota-derived metabolites Uro on colon fibroblasts upon IL-1 $\beta$ treatment as an in vitro inflammation model. Uro-A and Uro-B $(10 \mu \mathrm{M})$ inhibited $\mathrm{PGE}_{2}$ production $(85$ and $40 \%$, respectively) after IL-1 $\beta$ stimulation, whereas EA did not show any effect. Uro-A, but not Uro-B, down-regulated cyclo-oxygenase-2 (COX-2) and microsomal PGE synthase-1 (mPGES-1) mRNA expression and protein levels. Both Uro inhibited NF-кB translocation to nucleus. Slight but significant effects were found in the activation of mitogen-activated protein kinase (MAPK) pathways. Uro-A lowered c-Jun N-terminal kinase phosphorylation state, and both Uro inhibited p38 activation. No metabolites derived from Uro or EA were found in the cell media upon incubation of EA or Uro with the cells, and only traces of the compounds were found inside the cells. The present results suggest that Uro, mainly Uro-A, are the main compounds that are responsible for the pomegranate anti-inflammatory properties. The mechanism of action implicated seems to be via the inhibition of activation of NF- $\mathrm{B}$ and MAPK, down-regulation of COX-2 and mPGES-1 expressions, and consequently,via the reduction of $\mathrm{PGE}_{2}$ production. Taking into account that Uro did not enter the cells, a competitive binding for IL-1 $\beta$ membrane receptor cannot be discarded.

Cyclo-oxygenase-2: Microsomal PGE synthase-1: Polyphenol: CCD18-Co: Pomegranate

The incidence of inflammatory bowel diseases such as ulcerative colitis and Crohn's disease has risen in the last few years $^{(1)}$. An increment in cyclo-oxygenase-2 (COX-2)-derived PG synthesis has been described during acute and chronic intestinal inflammation, in particular, $\mathrm{PGE}_{2}$ synthesis. In addition, other $\mathrm{PGE}_{2}$ enzyme synthases have been described including the inducible microsomal PGE synthase-1 (mPGES-1), which has been functionally linked to COX-2 and is also overexpressed in some cancers ${ }^{(2,3)}$.

External stimuli such as inflammatory cytokines or UV irradiation induce the activation of NF- $\mathrm{KB}$ transcription factor by phosphorylation of I $\mathrm{B}$ that is degraded by the proteosome allowing NF- $\mathrm{BB}$ translocation to the nucleus ${ }^{(4)}$. Among the genes regulated by $\mathrm{NF}-\kappa \mathrm{B}$ are mPGES-1 and COX-2. In fact, $5^{\prime}$-promoter region of COX-2 contains two putative NF- $\mathrm{B}$-binding sites ${ }^{(5)}$. Other factors implicated in the inflammatory response are mitogen-activated protein kinases (MAPK). There are three major classes of MAPK, the extracellular signal-regulated kinase (ERK) and the two stress-activated protein kinase families c-jun $\mathrm{N}$-terminal kinase (JNK) and $\mathrm{p} 38$. MAPK regulate $\mathrm{NF}-\kappa \mathrm{B}$ activation by multiple mechanisms, and they are also implicated in COX-2 and mPGES-1 regulation ${ }^{(4,6)}$.

The increasing incidence of inflammatory bowel disease has been associated with changes in dietary pattern ${ }^{(7)}$. Although a clear relationship between fruit intake and inflammatory bowel disease has not been established yet, some fruits such as pomegranate have been shown to interact with different pathways involved in inflammation such as NF- $\mathrm{KB}$ activation and eicosanoid synthesis pathways and to influence cytokines and matrix metalloproteinase expression ${ }^{(8)}$. Some of the properties attributed to pomegranate are probably due to its polyphenol content ${ }^{(9,10)}$. Recently, the anti-inflammatory effects of a pomegranate extract and one of its major components, ellagic acid (EA), have been reported ${ }^{(11,12)}$. However, the compounds involved in these effects as well as the main mechanisms of action are not well understood.

Pomegranate ellagitannins (ET) are not absorbed but hydrolysed to produce EA, which is extensively metabolised by the gut microbiota to yield the hydroxy-dibenzopyranone

Abbreviations: COX-2, cyclo-oxygenase-2; EA, ellagic acid; ERK, extracellular signal-regulated kinase; ET, ellagitannins; JNK, c-Jun N-terminal kinase; MAPK, mitogen-activated protein kinase; mPGES-1, microsomal PGE synthase-1; Res, resveratrol; Uro, urolithin.

* Corresponding author: Mar Larrosa, fax +34 968 396213, email marlarrosa@cebas.csic.es 
<smiles>O=c1oc2cc(O)ccc2c2ccc(O)cc12</smiles>

Urolithin-A

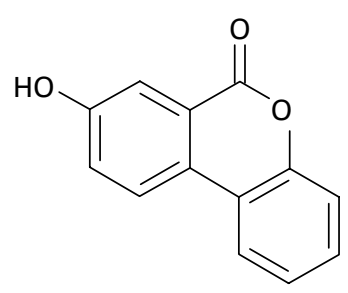

Urolithin-B
Fig. 1. Structures of the colonic microbiota ellagic acid-derived metabolites urolithin-A and urolithin-B.

derivatives urolithins (Uro, Fig. 1) ${ }^{(13-15)}$. Uro are bioavailable, and they can reach a high concentration at $\mu \mathrm{M}$ level as glucuronide derivatives in the bloodstream. Nevertheless, a large proportion of Uro reach distal parts of the colon in the aglycone form ${ }^{(16)}$. Despite polyphenols being metabolised in vivo, literature dealing with polyphenol-derived metabolites is scarce.

We previously studied the anti-inflammatory properties of a pomegranate extract and its main microbiota-derived metabolite Uro-A in a DSS colitis rat model ${ }^{(17)}$. Overall, the present results suggest that Uro-A could play a critical role in the antiinflammatory properties attributed to pomegranate consumption. These effects included the prevention of colon tissue damage and the decrease of inflammation markers (iNOS, COX-2, mPGES-1 and $\mathrm{PGE}_{2}$ in colonic mucosa). In addition, the $\mathrm{G} 1$ to $\mathrm{S}$ cell cycle pathway was up-regulated in both groups using microarray analyses. However, a favourable modulation of gut microbiota was also observed (increase of bifidobacteria and lactobacilli and decrease of enterobacteria). Therefore, the microbiota modulation could also be responsible for the observed effects. In addition, the individual effect of EA, Uro-A and Uro-B was not assessed.

In order to shed some light on the above, our main aim was to investigate whether Uro-A could act as an anti-inflammatory compound per se. To this purpose, Uro-A, Uro-B and EA were assayed in a colonic subepithelial fibroblast cell line to study their capacity to inhibit $\mathrm{PGE}_{2}$ production in response to inflammatory stimuli as well as to unravel the potential of the involved mechanisms of action.

\section{Materials and methods}

\section{Reagents}

EA, resveratrol (Res), quercetin and 3-(4,5-dimethylthiazol2-yl)-2,5-diphenyl tetrazolium bromide were purchased from Sigma-Aldrich (Steinheim, Germany). Uro-A (3,8-dihydroxy-6H-dibenzo $(b, d)$ pyran-6-one) and Uro-B (3-hydroxy$6 \mathrm{H}$-dibenzo $(b, d)$ pyran-6-one) were chemically synthesised by Kylolab S.A. (Murcia, Spain). IL-1 $\beta$ was purchased from Calbiochem (Milan, Italy). The specific inhibitors PD98059, SB203580, SP600125 and ammonium pyrrolidinedithiocarbamate (PDTC) were also obtained from Sigma-Aldrich.

\section{Cell culture and treatments}

The human normal colon fibroblast cell line CCD18-Co was obtained from the American Type Culture Collection
(ATCC number CLR-1459; Rockville, IN, USA), and was grown in minimal essential medium supplemented with $10 \%$ $\mathrm{v} / \mathrm{v}$ fetal bovine serum, $2 \mathrm{~mm}$ glutamine, $0 \cdot 1 \mathrm{~mm}$ non-essential amino acids, $1 \mathrm{~mm}$-sodium pyruvate, $0 \cdot 1 \mathrm{U} / \mathrm{ml}$ penicillin and $100 \mu \mathrm{g} / \mathrm{ml}$ streptomycin. Cells were maintained at $37^{\circ} \mathrm{C}$ under a $5 \% \mathrm{CO}_{2}$ and $95 \%$ air atmosphere at constant humidity. Stocks of EA, Uro-A, Uro-B, quercetin and Res were dissolved in DMSO, and were added to the culture media at the specified concentration. The final concentration of DMSO did not exceed $0.5 \%$. CCD18-Co cells were seeded and allowed to reach confluence, and subsequently, the medium was replaced with a fresh medium containing $0.1 \%$ fetal bovine serum $24 \mathrm{~h}$ after the inflammatory stimulus. Cells were then treated with EA and Uro-A and Uro-B (1 and $10 \mu \mathrm{M})$ and were co-stimulated with $1 \mathrm{ng} / \mathrm{ml}$ of IL-1 $\beta$ for different times specified below. CCD18-Co cells were used from population doubling level 28 to 35 for all the analyses.

\section{Cell viability}

Cells were seeded in 96-well plates and treated as described above. After $18 \mathrm{~h}$ of co-incubation with $10 \mu \mathrm{M}$ of EA, Uro-A or Uro-B and $1 \mathrm{ng} / \mathrm{ml}$ of IL-1 $\beta$, cells were washed three times with PBS, and the 3-(4,5-dimethylthiazol-2-yl)-2,5diphenyl tetrazolium bromide assay was performed as described by Morgan ${ }^{(18)}$.

\section{$P G E_{2}$ production assay}

$\mathrm{PGE}_{2}$ production was assayed as described by Larrosa et al. $^{(19)}$. Briefly, after serum starvation, cells were treated with EA, Uro-A, Uro-B $(1-10 \mu \mathrm{M})$ and with Res and quercetin $(1-10 \mu \mathrm{M})$ as anti-inflammatory polyphenol standards, and were co-stimulated with $1 \mathrm{ng} / \mathrm{ml}$ of IL-1 $\beta$ for $18 \mathrm{~h}$. Afterwards, media were recovered and $\mathrm{PGE}_{2}$ levels were measured using an immunoenzymatic method (EIA kit, Cayman Chemical, San Diego, CA, USA) according to the manufacturer's specifications. A dilution of 1:50 was used.

\section{Cyclo-oxygenase inhibitor screening assay}

Inhibitory activity of EA, Uro-A and Uro-B on COX-1 (bovine) and $\mathrm{COX}-2$ (human recombinant) enzymes was assayed using the colorimetric commercial kit (COX Inhibitor Screening Assay, Cayman Chemical) according to the manufacturer's protocol. Briefly, $150 \mu \mathrm{l}$ of assay buffer, $10 \mu \mathrm{l}$ of haem and $10 \mu \mathrm{l}$ of enzyme (either COX-1 or COX-2) were carefully mixed with $10 \mu \mathrm{l}$ of EA (1 and $10 \mu \mathrm{M})$, Uro-A or Uro-B $(10$ and $40 \mu \mathrm{M})$, and incubated at $25^{\circ} \mathrm{C}$ for $5 \mathrm{~min}$. Then, $20 \mu \mathrm{l}$ of arachidonic acid (substrate solution) were added and incubated at $25^{\circ} \mathrm{C}$ for $5 \mathrm{~min}$. Afterwards, absorbance was measured at $590 \mathrm{~nm}$ using a Fluostar galaxy microplate reader (BMG Labtech, Offenburg, Germany). Res $(30 \mu \mathrm{M})$ was used as a selective COX1/2 inhibitor positive control $^{(20,21)}$. The COX inhibitor screening assay directly measures $\mathrm{PGF} 2 \alpha$ produced by $\mathrm{SnCl}_{2}$ reduction of $\mathrm{COX}$ derived $\mathrm{PGH}_{2}$. The prostanoid product is quantified via enzyme immunoassay using a broadly specific antibody that binds to all the major PG compounds. All samples were expressed as percentage inhibition of initial activity. The assay was repeated twice. 


\section{RNA extraction}

Total RNA was isolated using an RNeasy mini kit (Qiagen, Hilden, Germany). RNA was eluted in RNAse-free water and stored at $-80^{\circ} \mathrm{C}$. RNA concentration and purity were checked using the Nanodrop spectrophotometer system (ND-1000 3.3 Nanodrop Technologies, Wilmington, DE, USA). Only samples with a ratio between 1.8 and 2.1 were used for quantitative PCR experiments.

\section{Quantitative real-time PCR}

Changes in the expression of COX-2 and mPGES- 1 genes were assessed by one-step quantitative PCR (Taqman system, Applied Biosystems, ABI, Madrid, Spain). Amplification was performed using a total reaction volume of $25 \mu \mathrm{l}$ in a MicroAmp Optical 96-well plate covered by optical adhesive covers, and using TaqMan Universal Master Mix. Primers and probes were selected from Assays-on-demand (ABI): PG-endoperoxide synthase-2 (COX-2), Hs00153133_m1; PGE synthase-1, Hs00610420_m1 (_m indicates an assay whose probe spans an exon junction and will not detect genomic DNA). The one-step quantitative PCR were run on the ABI 7500 system following the manufacturer's thermal conditions. The expression levels of target genes were normalised to the levels of glyceraldehyde-3-phosphate dehydrogenase (Hs99999905_m1) using a standard curve method for quantification. All assays were done in triplicate.

\section{Western blot analysis}

Cells were homogenised in cold RIPA buffer with protease inhibitor cocktail (Roche, Mannheim, Germany). Protein concentration was measured using Bradford's reagent, and $40 \mu \mathrm{g}$ of protein/lane was loaded. Antibodies that recognising phosphorylated and total p38 (phosphor-Tyr-182), JNK (phosphor-Thr-183/Tyr-185) and ERK-1/2 (phosphor-Thr185/Tyr-187) were purchased from Santa Cruz Biotechnology (Santa Cruz, CA, USA). COX-2 and mPGES-1 antibodies were purchased from Cayman Chemical (Ann Arbor, MI, USA). Glyceraldehyde-3-phosphate dehydrogenase antibody (Affinity BioReagents ${ }^{\mathrm{TM}}$, Golden, CO, USA) is routinely assayed for monitoring total protein load. Proteins were separated by 10-15\% SDS-PAGE, and were transferred to polyvinylidene difluoride membranes (GE Healthcare, Chalfont St Giles, Bucks, UK) by electroblotting. Proteins were detected using ECL plus detection system (GE Healthcare) according to the manufacturer's instructions. Density of the bands was detected using the ImageQuant TL v2005 (GE Healthcare). The Western blot assays were done in triplicate for each treatment.

\section{$N F-\kappa B$ nuclear translocation assay}

$\mathrm{NF}-\kappa \mathrm{B}$ activation was determined with the ELISA TransAM ${ }^{\mathrm{TM}}$ NF- $\kappa$ B p65 kit (Active Motif, Rixensart, Belgium), which specifically measures the amount of NF- $\mathrm{B}$ p65 bound to its consensus binding site ( $5^{\prime}$ GGGACTTTCC $\left.3^{\prime}\right)$. Nuclear extracts were prepared according to the procedure of Oh \& Lee ${ }^{(22)}$. Protein concentration in the nuclear extracts was determined using Bradford's method. Jurkat nuclear extract $(2.5 \mu \mathrm{g})$ was used as a positive control.
Cell-based extracellular signal-regulated kinases 1/2, c-Jun $N$-terminal kinase and p38 mitogen-activated protein kinase phosphorylation ELISA

Changes in the relative amount of ERK1/2, JNK and p38 phosphorylation state in treated CCD18-Co cells were determined using a RayBio Cell-Based ERK1/2, JNK and p38 MAPK ELISA kit (RayBiotech, Inc., Norcross, GA, USA) following the manufacturer's protocol. Briefly, after $24 \mathrm{~h}$ of serum starvation, cells were treated with EA, Uro-A or Uro-B $(10 \mu \mathrm{M})$, and were co-stimulated with $1 \mathrm{ng} / \mathrm{ml}$ of IL-1 $\beta$ for $30 \mathrm{~min}, 2$ and $4 \mathrm{~h}$, with or without pre-treatment with the specific inhibitors. The ERK-1/2 inhibitor PD98059 (25 $\mu \mathrm{M})$, the JNK inhibitor SP600125 $(10 \mu \mathrm{M})$ and the selective p38 MAPK inhibitor SB203580 (25 $\mu \mathrm{M})$ were added $1 \mathrm{~h}$ before the application of the inflammatory stimulus. Media were discarded at different time points, and the cells were washed and fixed for $20 \mathrm{~min}$ at room temperature followed by $1 \mathrm{~h}$ blocking step. Anti-phospho-ERK1/2, phospho-JNK or phospho-p38 antibodies or anti-ERK1/2, JNK or p38 antibodies were added to the corresponding wells and incubated for $2 \mathrm{~h}$ at room temperature. The cells were then washed and incubated with HRP-conjugated anti-mouse IgG for $1 \mathrm{~h}$. After washing, the substrate 3,3',5,5'-tetramethylbenzidine was added and incubated for $30 \mathrm{~min}$ at room temperature. Absorbance was measured at $450 \mathrm{~nm}$ using a Fluostar galaxy microplate reader (BMG Labtech).

\section{Cellular uptake}

CCD18-Co cells were seeded at $44 \times 10^{3} \mathrm{~cm}^{2}$ density in $9 \mathrm{~cm}$ diameter plates with EMEM without phenol red and with the supplements specified above. The presence of metabolites excreted into the medium as well as those located inside the cells was explored. Cells were treated with Uro-A, Uro-B and EA $(10 \mu \mathrm{M})$, and were co-stimulated or not with $1 \mathrm{ng} / \mathrm{ml}$ of IL-1 $\beta$ in order to check whether inflammation conditions could affect cell metabolism. After $18 \mathrm{~h}$, the medium was recovered and processed as described by Larrosa et al. ${ }^{(23)}$. Aliquots of $5 \mu \mathrm{l}$ of media and cell extract samples were analysed by liquid chromatography-MS/MS.

\section{Liquid chromatography-MS/MS analyses}

The HPLC-DAD system (1200 series; Agilent Technologies, Waldbronn, Germany) was equipped with an HTC Ultra mass detector in series (Bruker Daltonics, Bremen, Germany). The mass detector was an ion trap mass spectrometer equipped with an electrospray ionisation (capillary voltage, $4 \mathrm{kV}$; nebuliser $15 \mathrm{psi}$; dry gas $5 \mathrm{l} / \mathrm{min}$; dry temperature, $350^{\circ} \mathrm{C}$ ) system. Mass scan and MS/MS daughter spectra were measured from $\mathrm{m} / \mathrm{z}, 100$ to 600 using the Ultra scan mode $(26000 \mathrm{~m} / \mathrm{z}$ per second). Collision-induced fragmentation experiments were performed in the ion trap using $\mathrm{He}$ as the collision gas, and the collision energy was set at $50 \%$. MS data were acquired in the negative ionisation mode. Chromatographic separations of samples were carried out on a $150 \times 0.5 \mathrm{~mm}$ internal diameter, $5 \mu \mathrm{m}$, reverse phase SB C18 Zorbax column (Agilent) using water-formic acid (99:1, v/v) (A) and acetonitrile (B) as the mobile phases at a flow rate of $10 \mu \mathrm{l} / \mathrm{min}$. The gradient started with $1 \% \mathrm{~B}$ in $\mathrm{A}$ to reach $60 \% \mathrm{~B}$ at $30 \mathrm{~min}, 90 \% \mathrm{~B}$ 
at $30 \mathrm{~min}$ for $5 \mathrm{~min}$ and returned to the initial conditions (1\% B). UV chromatograms were recorded at 280,305 and $360 \mathrm{~nm}$.

Identification and quantification of Uro-derived and ellagic acid-derived metabolites, Uro and EA were performed as described by Cerdá et al. ${ }^{(24)}$.

\section{Statistical analysis}

All analyses were carried out using the SPSS ${ }^{\text {тм }} 14.0$ software (SPSS Inc, Chicago, IL, USA). All data are expressed as the mean values and standard deviations of independent measurements. Statistical significance was determined by Student's $t$ test using $P<0.05$ as the level of significance.

\section{Results}

\section{$P G E_{2}$ production}

To test whether EA, Uro-A and Uro-B could modulate $\mathrm{PGE}_{2}$ synthesis, the production of $\mathrm{PGE}_{2}$ by CCD18-Co cells was measured upon stimulation with $1 \mathrm{ng} / \mathrm{ml}$ of IL-1 $\beta$ for $18 \mathrm{~h}$. IL-1 $\beta$ produced a significant increase (30-fold) in $\mathrm{PGE}_{2}$ levels $(P<0 \cdot 05$; Fig. 2). Co-treatment with Uro-A (1 and $10 \mu \mathrm{M})$ significantly $(P<0.05)$ decreased $\mathrm{PGE}_{2}$ levels in a dose-dependent manner (2.6-fold and 8-fold, respectively). Uro-B significantly lowered $\mathrm{PGE}_{2}$ production by 1.5 -fold at $10 \mu \mathrm{M}$, while EA had no effect at any concentration tested (Fig. 2). Res $(10 \mu \mathrm{M})$ and quercetin $(10 \mu \mathrm{M})$, used as positive controls, also significantly inhibited $\mathrm{PGE}_{2}$ secretion approximately to the same extent as Uro-A $(10 \mu \mathrm{M})$ inhibited it. None of the compounds tested affected the cells growth or caused cytotoxicity measured by the 3-(4,5-dimethylthiazol-2-yl)-2, 5-diphenyl tetrazolium bromide method (data not shown).

\section{Cyclo-oxygenase-1 and cyclo-oxygenase-2 inhibition assay}

Direct inhibition of COX-1 and COX-2 activities by EA ( 1 and $10 \mu \mathrm{M})$, and Uro-A and Uro-B (10 and $40 \mu \mathrm{M})$ was assessed

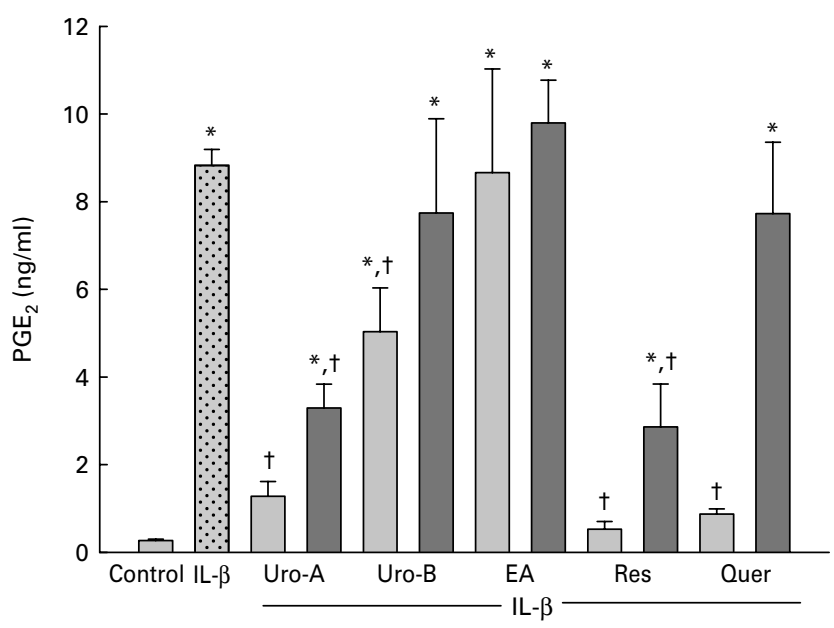

Fig. 2. $P G E_{2}$ levels in CCD18-Co cells treated with Urolithin (Uro)-A, Uro-B, ellagic acid (EA), resveratrol (Res) and quercetin (Quer; $1-10 \mu \mathrm{M}$ ) and co-stimulated with IL-1 $\beta(1 \mathrm{ng} / \mathrm{ml})$ for $18 \mathrm{~h}$. Each bar represents the mean values and standard deviations ( $n 3$ ). ${ }^{*}$ Mean values were significantly different to the control $(P<0.05)$. † Mean values were significantly different over IL-1 $\beta$ treatment $(P<0.05)$. $\square, 10 \mu \mathrm{M} ; \square, 1 \mu \mathrm{M}$. with a commercially available COX inhibitory screening assay using purified COX-1 (ovine) and COX-2 (human recombinant) enzymes. Neither EA nor Uro-A nor Uro-B inhibited COX-1 or COX-2- activity at any concentration tested, while Res $(30 \mu \mathrm{M})$ that was used as a positive control showed significant $(P<0.01)$ inhibitory effects, 96.44 (SD 1.90) on COX-1 and 57.82 (SD 8.48) COX-2 enzyme activities (results expressed as \% inhibition (SD)).
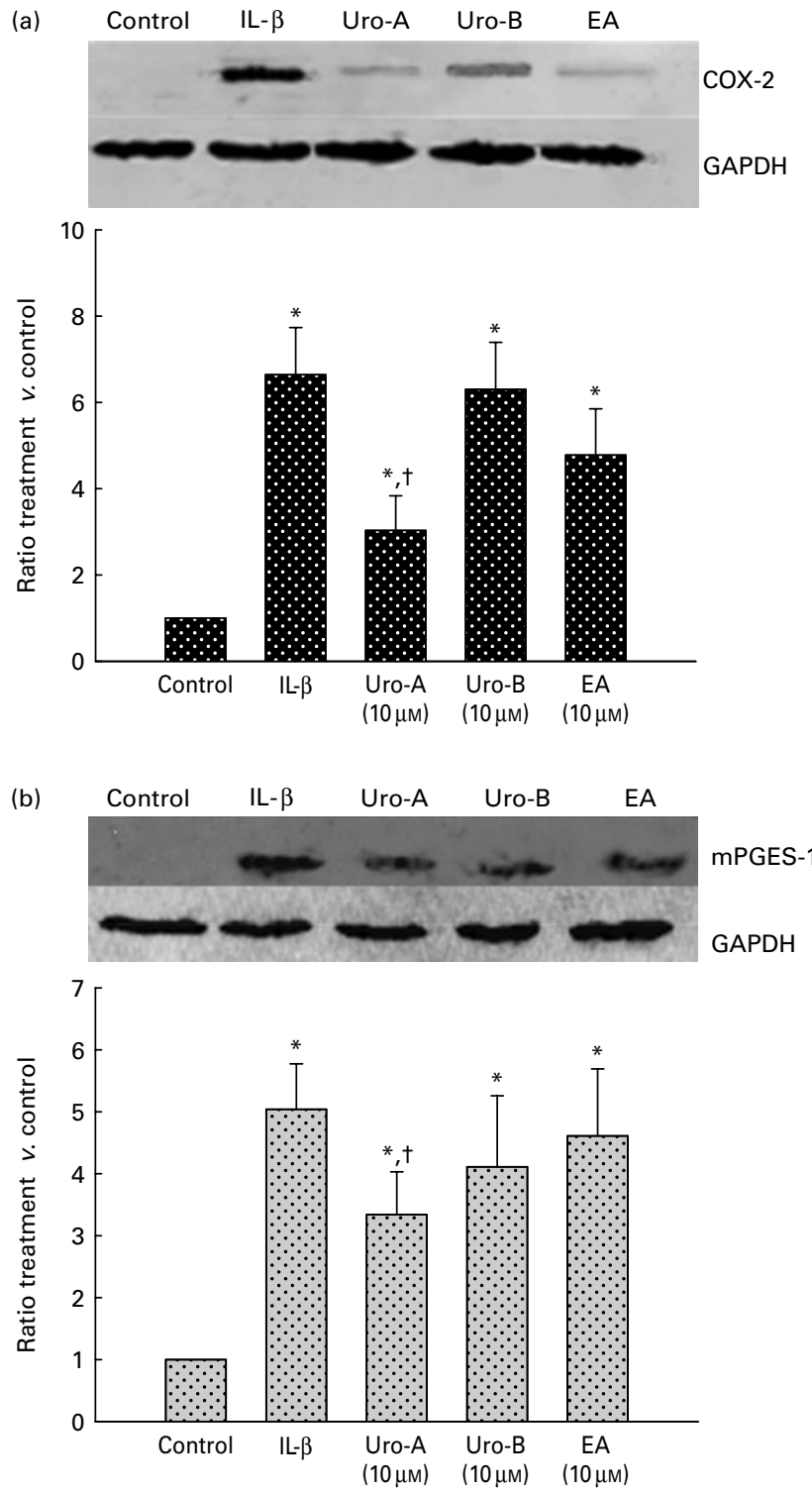

Fig. 3. Western blot analysis of cyclo-oxygenase-2 (COX-2 (a)) and microsomal PGE synthase-1 (mPGES-1 (b)). Protein was detected in CCD18-Co cells stimulated with $1 \mathrm{ng} / \mathrm{ml}$ of $\mathrm{IL}-1 \beta$ and treated with $10 \mu \mathrm{M}$ of Urolithin (Uro)-A, Uro-B or ellagic acid (EA) for $18 \mathrm{~h}$. ${ }^{*}$ Mean values were significantly different in protein levels to the control $(P<0.05)$. † Mean values were significantly different in protein levels over IL- $\beta$ treatment $(P<0.05)$. Results are expressed as mean values and standard deviations $(n 3)$. The Western blot image illustrates the expression change, and the densitometric values are the mean of three independent experiments. GAPDH, glyceraldehyde3-phosphate dehydrogenase. 
Cyclo-oxygenase-2 and microsomal PGE synthase-1 protein and $m R N A$ levels

As PGE $_{2}$ levels in an inflammation state are determined by two inducible enzymes, COX-2 and mPGES-1, their expression was analysed by Western blot analysis and quantitative PCR in CCD18-Co cells stimulated with IL-1 $\beta$ and treated simultaneously with EA, Uro-A and Uro-B.

Firstly, changes in protein levels of both COX-2 and mPGES-1 were assessed. COX-2 and mPGES-1 protein expressions were undetectable in control cells, and were noticeably induced (6- and 5-fold, respectively) in cells treated with IL-1 $\beta$ for $18 \mathrm{~h}$ (Fig. 3). Uro-A $(10 \mu \mathrm{M})$ significantly $(P<0.05)$ lowered COX-2 (3-fold) and mPGES-1 (2-fold) proteins. Neither COX-2 nor mPGES-1 protein levels were changed upon exposure of cells to EA $(10 \mu \mathrm{M})$ or Uro-B $(10 \mu \mathrm{M})$.

To validate the present results at the mRNA level, COX-2 and mPGES-1 mRNA time-induced expressions by $1 \mathrm{ng} / \mathrm{ml}$ IL-1 $\beta$ treatment were analysed. The mRNA levels of COX-2 and mPGES-1 in $1 \mathrm{ng} / \mathrm{ml}$ IL- $1 \beta$-treated cells reached a maximum of 200 -fold at $4 \mathrm{~h}$ and 20 -fold at $8 \mathrm{~h}$ of incubation,
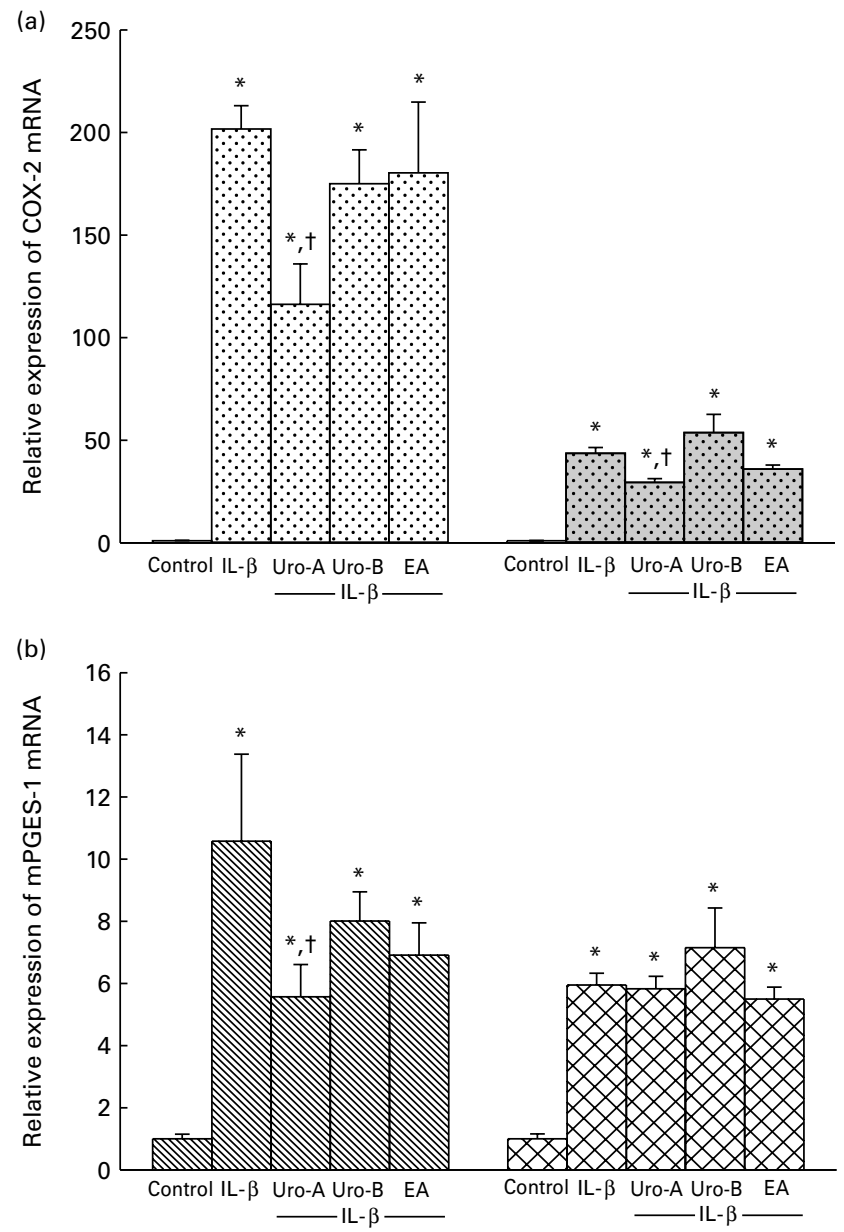

Fig. 4. Quantitative PCR analysis of cyclo-oxygenase-2 (COX-2 (a)) and microsomal PGE synthase-1 (mPGES-1 (b)) expressions in CCD18-Co cells. Data were studied following normalisation to glyceraldehyde-3-phosphate dehydrogenase (GAPDH). Results are expressed as mean values and standard deviations $(n 6)$. * Mean values were significantly different to the control $(P<0.05)$. † Mean values were significantly different over IL-1 $\beta$ treatment $(P<0.05)$. Uro, urolithin; EA, ellagic acid. (a) $⿴$, COX-2 $4 \mathrm{~h}$; 圈, COX-2

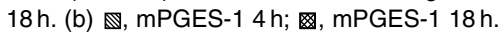

respectively (data not shown), with the increment being less marked at $18 \mathrm{~h}$ (50-fold for COX-2 and 6-fold for mPGES-1; Fig. 4). Accordingly, in subsequent experiments, COX-2 and of mPGES-1 mRNA levels were measured at 4 and $18 \mathrm{~h}$ of treatment. Treatment with Uro-A $(10 \mu \mathrm{M})$ significantly $(P<0.05)$ decreased mRNA levels of COX-2 at 4 and $18 \mathrm{~h}$ (Fig. 4(a)) and mPGES-1 at $4 \mathrm{~h}$ but not at $18 \mathrm{~h}$ (Fig. 4(b)). Cells treated with Uro-B and EA exhibited mRNA levels of COX-2 and mPGES-1 that were similar to those exhibited by the cells treated with IL-1 $\beta$ (Fig. 4).

\section{NF- $\kappa$ B p65 DNA-binding assay}

In a previous screening, treatment with $1 \mathrm{ng} / \mathrm{ml}$ of IL-1 $\beta$ at $30 \mathrm{~min}, 2$ and $4 \mathrm{~h}$ resulted in the nuclear translocation of p65 $\mathrm{NF}-\kappa \mathrm{B}$ and binding to its DNA consensus sequence at 2 and $4 \mathrm{~h}$ but not at $30 \mathrm{~min}$ (data not shown). Subsequent experiments were done at 2 and $4 \mathrm{~h}$ (Fig. 5). Treatments with Uro-A $(10 \mu \mathrm{M})$ and Uro-B $(10 \mu \mathrm{M})$ significantly inhibited $(P<0.05)$ p65-binding activity at both times. EA did not produce any effect on NF-кB (Fig. 5).

Involvement of $N F-\kappa B$ in cyclo-oxygenase-2 and microsomal $P G E$ synthase-1 expressions

Cells were treated for $1 \mathrm{~h}$ with the NF-кB inhibitor PDTC $(100 \mu \mathrm{M})$ before IL-1 $\beta$ stimulation. COX-2 and mPGES-1 expressions were checked after $18 \mathrm{~h}$ by Western blot analysis. Treatment with PDTC significantly decreased both mPGES-1 and COX-2 expressions (Fig. 6), confirming the participation of NF- $\mathrm{KB}$ in the regulation of COX-2 and mPGES-1 expressions.

Phosphorylation status of extracellular signal-regulated kinase, $c$-Jun $N$-terminal kinase and p38 signalling in CCD18-Co cells

Since MAPK pathways are involved in IL-1 $\beta$-induced inflammation, the potential influence of MAPK signalling pathways

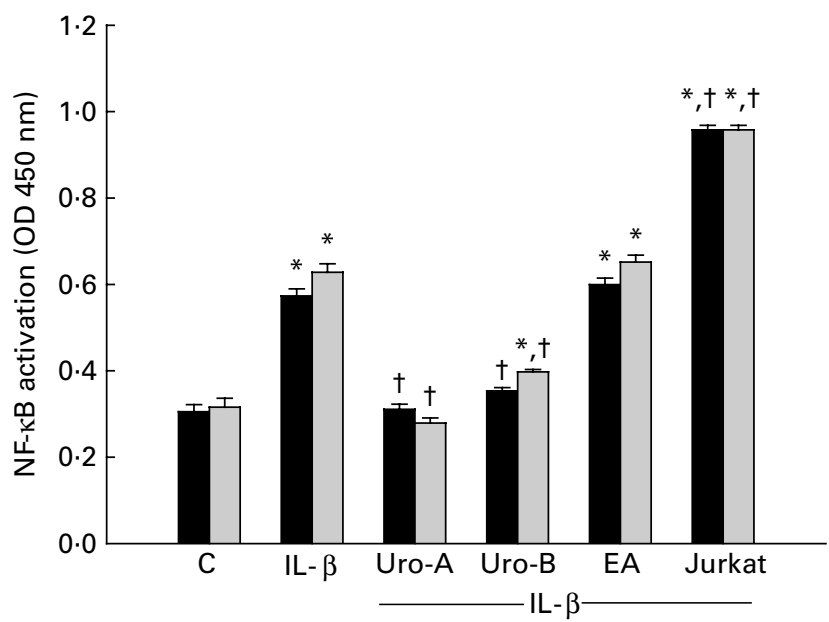

Fig. 5. Effect of ellagic acid (EA), urolithin (Uro)-A and Uro-B on the activation of NF-kB p65. *Mean values were significantly different to the control $(P<0.05)$. † Mean values were significantly different over IL-1 $\beta$ treatment $(P<0.05)$. OD, optical density; C, control. $\square, 2 \mathrm{~h} ; \square, 4 \mathrm{~h}$. 

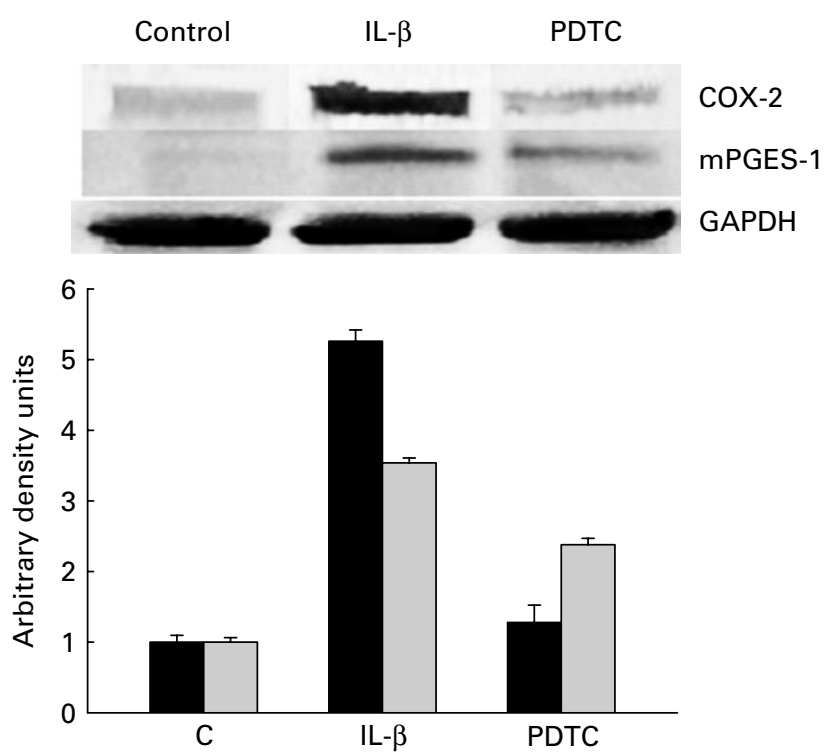

Fig. 6. Inhibition of NF- $\mathrm{kB}$ activation prevents cyclo-oxygenase-2 (COX-2) and microsomal PGE synthase-1 (mPGES-1) expression in CCD18-Co cells stimulated with IL-1 $\beta$. Pyrrolidinedithiocarbamate (PDTC), NF- $\mathrm{kB}$ inhibitor. The Western blot image illustrates the expression change, and the densitometric values are the mean of three independent experiments. GAPDH, glyceraldehyde-3-phosphate dehydrogenase. $\mathbf{E}$, COX-2; $\square$, mPGES-1.

was studied upon Uro-A, Uro-B and EA treatment. Both total and phosphorylated forms of ERK1/2, JNK and p38 in cells stimulated with IL-1 $\beta$ were measured at $30 \mathrm{~min}, 2$ and $4 \mathrm{~h}$ using cell-based ELISA. Stimulation of cells with $1 \mathrm{ng} / \mathrm{ml}$ of IL-1 $\beta$ produced a significant increase in the phosphorylation status (activation) of the ERK1/2, JNK, and p38 kinases at $30 \mathrm{~min}$ (Fig. 7). However, this effect was not maintained at 2 and $4 \mathrm{~h}$ for the ERK1/2 and JNK and only at $2 \mathrm{~h}$ for p38 (results not shown). Therefore, the influence of EA, Uro-A and Uro-B on these kinases was tested at $30 \mathrm{~min}$. When cells were stimulated with IL- $1 \beta$ and co-treated with Uro-A $(10 \mu \mathrm{M})$, the phosphorylation state of JNK and p38 kinases was slightly but significantly lowered $(P<0.05)$, while no effect was observed on the ERK1/2 pathway. Uro-B $(10 \mu \mathrm{M})$ significantly attenuated p38 activation, whereas no effect $(P<0.05)$ was observed on JNK pathway (Fig. 7). EA had no effect on ERK1/2 and p38 kinases and it significantly increased the activation of JNK. Pre-incubation for $1 \mathrm{~h}$ with the specific inhibitors PD98059 $(25 \mu \mathrm{M})$, SB203580 $(25 \mu \mathrm{M})$ and SP600125 $(10 \mu \mathrm{M})$ as positive controls significantly reduced $(P<0.05)$ the activation of ERK1/2, p38 and JNK, respectively (Fig. 7). The results obtained with the ELISA kits were further confirmed by Western blot analysis (Fig. 8). Therefore, the slight but statistically significant changes in the phosphorylation state of MAPK indicated their involvement in the activation of the transcription factor NF- $\mathrm{B}$.

\section{Cellular uptake}

Cell media and cell extract samples were analysed by LCMS/MS to explore cell metabolism of EA, Uro-A and Uro-B under normal and inflammation conditions. No metabolites derived from EA, Uro-A or Uro-B were detected in cell media or in cell extracts in both normal and inflammation conditions, and incubation times were assayed. Trace amounts of Uro-A (6.4 pM), Uro-B (3.33 pM) and EA (0.68 pM) were found in the cell extracts.

\section{Discussion}

Antioxidant, anti-inflammatory and anticancer properties have been attributed to pomegranate and other ellagitannin-containing foods. Most studies that demonstrated pomegranate properties have been conducted with juices or extracts that

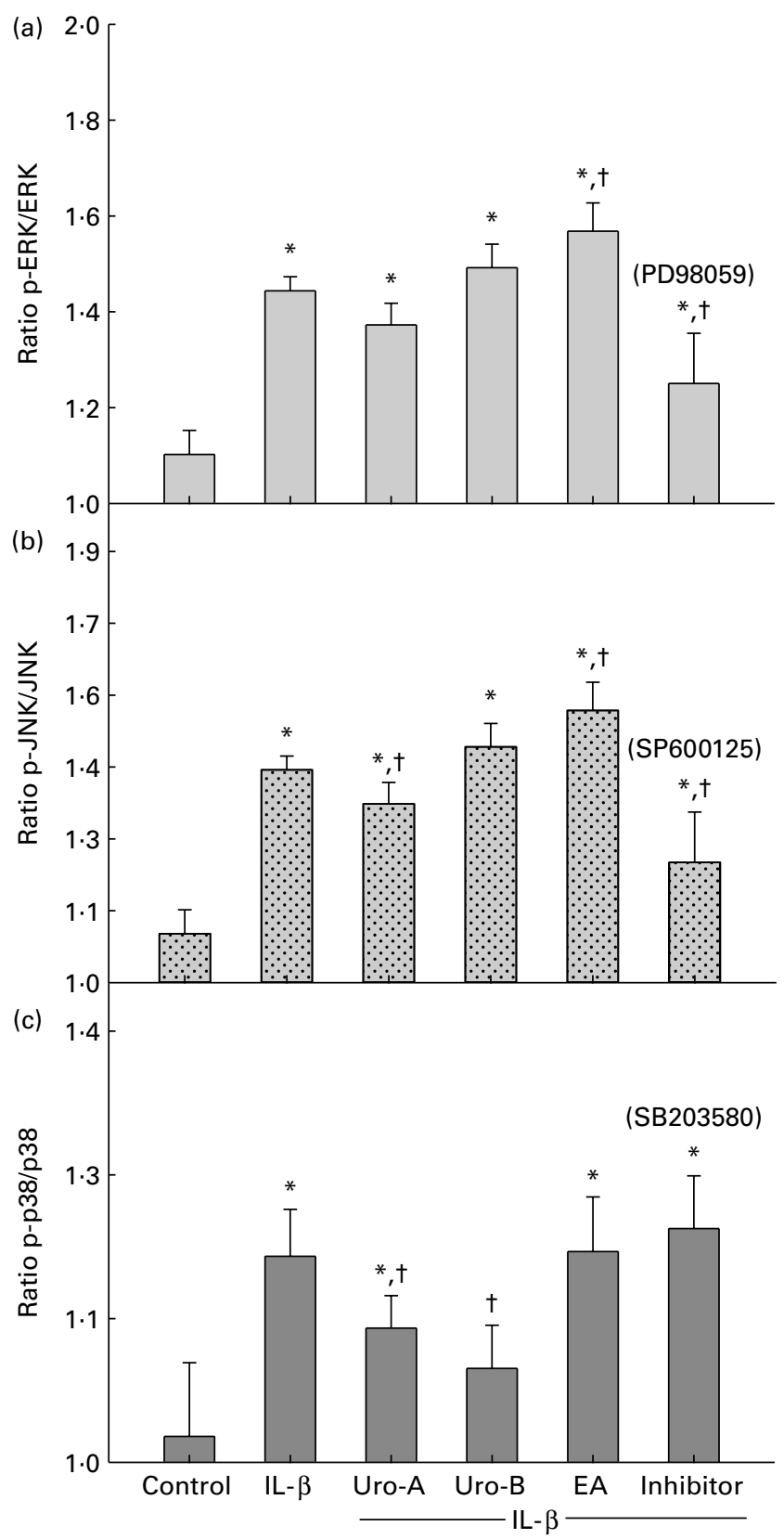

Fig. 7. Effect of ellagic acid (EA), Urolithin (Uro)-A and Uro-B on mitogenactivated protein kinase activation. Extracellular signal-regulated kinase (ERK1/2 (a)), c-Jun N-terminal kinase (JNK (b)) and p38 (c). * Mean values were significantly different to the control $(P<0.05)$. †Mean values were significantly different over IL-1 $\beta$ treatment $(P<0.05)$. All experiments were carried out in triplicate. Plotted values represent mean values and standard deviations. 

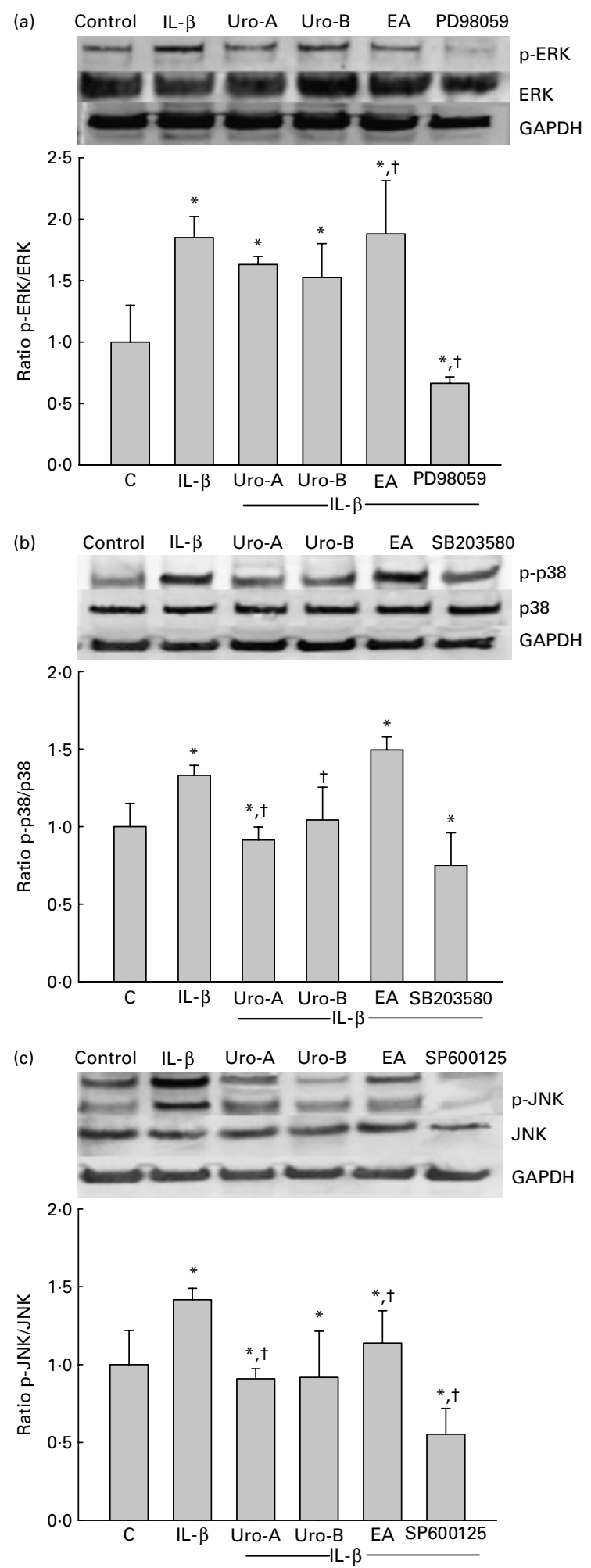

Fig. 8. Western blot analysis of the effect of ellagic acid (EA), Urolithin (Uro)-A and Uro-B on mitogen-activated protein kinase activation. Extracellular signal-regulated kinase (ERK1/2 (a)), c-Jun N-terminal kinase (JNK (b)) and p38 (c). * Mean values were significantly different to the control $(P<0.05)$. † Mean values were significantly different over IL-1 $\beta$ treatment $(P<0.05)$. Results are expressed as mean values and standard deviations $(n 3)$. The Western blot image illustrates the expression change, and the densitometric values are the mean of three independent experiments.

contain simple sugars, amino acids, fibre, sterols and a large amount of polyphenols including anthocyanins, flavones, flavonols and mainly $\mathrm{ET}^{(8)}$. It has been suggested that polyphenols may be partly responsible for the beneficial activities attributed to pomegranate ${ }^{(25,26)}$. However, these in vitro studies did not take into account the bioavailability of pomegranate polyphenols. Pomegranate ET are not absorbed as such, but they are hydrolysed to EA and further metabolised by colon microbiota to yield Uro ${ }^{(13-15,24,27)}$. Uro are bioavailable, and can reach significant concentrations as glucuronide conjugates in plasma and urine, whereas high Uro aglycone concentrations (above $100 \mu \mathrm{M}$ ) can reach the colon ${ }^{(28,16)}$. Previous results in our research group suggested an in vivo anti-inflammatory effect of the pomegranate-derived metabolite Uro- $\mathrm{A}^{(17)}$. However, other potential anti-inflammatory actions mediated by gut microbiota could also be responsible for the effects observed. In this context, the individual role of pomegranate metabolites in colon and their mechanism of action were not deeply studied.

Intestinal fibroblasts play a pivotal role by influencing epithelial function, and amplifying or reducing the inflammatory response by secreting mediators such as $\mathrm{PGE}_{2}$ and metalloproteinases ${ }^{(29,30)}$. The present results show that treatment of colonic fibroblast with IL- $1 \beta$ increases PGE $_{2}$ levels, and that this response is partially attenuated by Uro-A and Uro-B but not by EA. Therefore, we confirmed that Uro-A was able to inhibit $\mathrm{PGE}_{2}$ synthesis, which was in accordance with our previous findings in DSS-treated rats upon oral administration with Uro-A ${ }^{(17)}$.

We next assessed the effect of Uro-A, Uro-B and EA on the activity of COX-1 and COX-2 enzymes to check whether a direct inhibition of these enzymes was the reason for the attenuated $\mathrm{PGE}_{2}$ production. None of the compounds showed inhibitory enzyme activity. There is no literature about the inhibition of COX-1 or COX-2 activity by Uro. Regarding $\mathrm{EA}$, the present results are in agreement with the lack of inhibitory properties of EA for COX-2 activity or $\mathrm{PGE}_{2}$ production $^{(31,32)}$. This suggests that others instead of EA are responsible for pomegranate COX-2 inhibitory activity, since a recent report has demonstrated that metabolites that become bioavailable in plasma after pomegranate fruit extract ingestion are able to inhibit COX-2 activity ${ }^{(11)}$. $\mathrm{PGE}_{2}$ production in inflammation status is determined by COX-2 and mPGES-1 enzymes. A number of studies have reported that cells overexpressing both COX-2 and mPGES-1 enzymes produce more $\mathrm{PGE}_{2}$, which suggests that mPGES-1 inhibition could improve the adverse effects of $\mathrm{PGE}_{2}$ production ${ }^{(33)}$. Thus, we further studied protein and mRNA expression of these enzymes. Uro-A reduced COX-2 and mPGES-1 expressions at both mRNA and protein levels. However, the doses assayed for Uro-B and EA had no effect on either COX-2 or mPGES-1 expression. Inhibition of COX-2 expression by pomegranate extract and juice in vitro and after topical application has been reported previously ${ }^{(26,34)}$. Probably, in these studies, ET are the responsible fraction for the observed in inhibition COX-2 expression as the assay conditions were not physiological (i.e. absence of metabolism, gut microbiota, etc.). The results presented here regarding the down-regulation of COX-2 and mPGES-1 expressions match with our previous results in inflamed colonic mucosa of rats fed with pomegranate extract or Uro- $\mathrm{A}^{(17)}$. 
Stimulation of cells with the inflammatory cytokine IL-1 $\beta$ led to an activation of the pathways of NF- $\mathrm{BB}$ transcription factor and MAPK that mediate COX-2 induction in many cell types ${ }^{(35-38)}$. The treatment with IL-1 $\beta$ activated NF-кB and all the three classes of MAPK (ERK, JNK and p38 MAPK). The present results show that Uro-A and Uro-B inhibited NF- $\mathrm{BB}$ activation by preventing the union of the p65 subunit to its consensus binding site, suggesting that inhibition of COX-2 and mPGES-1 expression and therefore $\mathrm{PGE}_{2}$ could be mediated by NF- $\mathrm{B}$ inhibition. A slight inhibition of p38 MAPK activation was detected upon incubation with both Uro-A and Uro-B. In the case of JNK, Uro-A displayed a slight inhibition, whereas no effect was observed with Uro-B treatment. In addition, neither Uro-A nor Uro-B lowered the phosphorylation status of ERK1/2. These results are in contrast with previous findings showing a large decrease in the activated form $\mathrm{p}$-ERK1/2 after incubation with a mixture of Uro and EA in Caco-2 cells for $72 \mathrm{~h}^{(39)}$. However, it should be highlighted that the divergence in results could be due to the cell line assayed (cancer epithelial Caco-2 $v$. normal fibroblastic CCD18-Co), incubation time $(72 v .18 \mathrm{~h})$ and different doses assayed (mixture of $40 \mu \mathrm{M}$ of Uro-A and Uro-B and $10 \mu \mathrm{M}$ EA $v .10 \mu \mathrm{M}$ of each individual compound in the present study) as well as due to the lack of inflammatory stimuli in the study of González-Sarrías et al. ${ }^{(39)}$. We cannot rule out that the effect observed in the previous study in Caco- 2 cells was due to the $40 \mu \mathrm{M}$ Uro-A treatment.

On the contrary, EA did not attenuate NF- $\kappa$ B or MAPK activation in accordance with the lack of anti-inflammatory activity. These data are in agreement with the study done by Masamune et al. ${ }^{(40)}$, who reported the lack of inhibition of $N F-\kappa B$ translocation to nucleus in IL-1 $\beta$-treated stellate cells upon incubation with EA. However, the inhibition of MAPK activation was observed in that study ${ }^{(40)}$. These contradictory data regarding MAPK activation could be due to the different MAPK activation patterns depending on the cell type ${ }^{(4)}$. A slight increase in ERK phosphorylation was detected in cells treated with EA over cells treated with IL-1 $\beta$ (Fig. 7(a)). This activation could be due to $\mathrm{PGE}_{2}$ accumulation, which can activate the ERK pathway in several cell lines ${ }^{(41)}$ and whose production was not inhibited by EA (Fig. 2).

The present results show that the anti-inflammatory effects of Uro-A and Uro-B are mediated by the inhibition of NF- $\mathrm{B}$ transcription factor, despite the effects that were observed on the inhibition of MAPK activation being slight. For this reason, the results were confirmed by two experimental techniques (ELISA and Western blot analysis). However, the role of NF- $\kappa \mathrm{B}$ and MAPK in the induction of COX-2 expression and $\mathrm{PGE}_{2}$ production after IL-1 $\beta$ treatment is not yet fully understood. For instance, IL- $1 \beta$ stimulation of cardiac myocytes or smooth tracheal cells led to an increased $\mathrm{PGE}_{2}$ production and COX-2 expression that was mediated by MAPK (p38 and ERK) and NF- $\mathrm{KB}$ activation but through independent pathways $^{(37,38)}$. However, induction of COX-2 expression required the activation of both JNK and p38 MAPK pathways in renal mesangial cells ${ }^{(36)}$. For these reasons, further research is needed to elucidate the mechanisms implicated in the antiinflammatory effects of these compounds using other cell models and inflammatory stimuli. In addition, other promoters involved in the transcriptional activation of COX-2 such as $\mathrm{C} / \mathrm{EBP}$ or $\mathrm{CRE} / \mathrm{ATF}^{(42,43)}$, the transcription factor Egr-1 that contributes to mPGES-1 expression ${ }^{(44)}$, the cytosolic phospholipase $\mathrm{A} 2$ or the protein kinase $\mathrm{A}$ that has also been described in the IL-1 $\beta$-mediated $\mathrm{PGE}_{2}$ synthesis ${ }^{(45)}$, should be studied.

The present results also suggest a receptor-mediated antiinflammatory effect as no metabolites were found in cell media or cell extracts upon incubation Uro-A or Uro-B using this cell line. Substantial uptake and cell metabolism have been reported recently in Caco- 2 cells upon incubation with either Uro-A or Uro- $\mathrm{B}^{(16)}$. It is of note that fibroblasts are metabolically less active than epithelial cells. In addition, 'normal' over 'cancerous' phenotype could also have a great influence ${ }^{(46)}$. In the present study, a trace amount of Uro-A was detected in the cell extracts $(6.4 \mathrm{pM})$, although this could be due to contamination of cell media. Whether the anti-inflammatory effect was due to a competitive binding of Uro with IL- $1 \beta$ receptor or to the very low Uro concentration detected inside the cells remains unanswered.

In summary, Uro-A decreased $\mathrm{PGE}_{2}$ production by downregulating $\mathrm{COX}-2$ and $\mathrm{mPGES}-1$ expressions, preventing $\mathrm{NF}-\kappa \mathrm{B}$ activation and decreasing the activated forms of $\mathrm{p} 38$ MAPK and JNK. Uro-B was able to inhibit the activation of NF- $\kappa$ B and p38 MAPK. However, attenuation of $\mathrm{PGE}_{2}$ levels was lower than in the case of Uro-A, and no effect on COX-2 and mPGES-1 at the assayed doses was observed. No anti-inflammatory effects were observed for EA under these assay conditions.

Further research into the possible synergy between both Uro and the assay of other inflammation models could help to discern whether the consumption of ET-containing foods (as Uro precursors) could improve our health status by preventing the incidence of inflammation-related diseases, especially those affecting the colon where Uro can reach high concentrations.

\section{Acknowledgements}

The present work was supported by the Projects CICYTBFU2007-60576 and Consolider Ingenio 2010, CSD200700063 (Fun- $C$-Food). A. G.-S. is the holder of a FPI predoctoral grant from MICINN (Spain). M. L. is the holder of a postdoctoral grant from MICINN. A. G.-S. carried out the laboratory experiments: cell culture, quantitative PCR, ELISA and western blot analysis. M. L. carried out the $\mathrm{PGE}_{2}$ measurements, and contributed to the design of the experiments, analysis of data and drafting of the manuscript. F. A. T.-B. and P. D. contributed to drafting and revising of the manuscript critically. J. C. E. contributed to the conception and design of the experiments, interpretation of data, drafting of the manuscript, revising and final approval. The authors declare that there is no conflict of interest.

\section{References}

1. Rubio CA \& Befrits R (2008) Colorectal cancer in Crohn's disease review of a 56-year experience in Karolinska Institute University Hospital. J Environ Pathol Toxicol 27, 257-266.

2. Rask K, Zhu Y, Wang W, et al. (2006) Ovarian epithelial cancer: a role for $\mathrm{PGE}_{2}$-synthesis and signalling in malignant transformation and progression. Mol Cancer 5, 62.

3. Wang HW, Hsueh CT, Lin CF, et al. (2006) Clinical implications of microsomal prostaglandin $\mathrm{E}$ synthase-1 overexpression in 
human non-small-cell lung cancer. Ann Surg Oncol 13, $1224-1234$.

4. Surh YJ, Chun KS, Cha HH, et al. (2001) Molecular mechanisms underlying chemopreventive activities of anti-inflammatory phytochemicals: down-regulation of COX-2 and iNOS through suppression of NF-kappa B activation. Mutat Res 1, 480-481, 243-268.

5. Catley MC, Chivers JE, Cambridge LM, et al. (2003) IL-1betadependent activation of NF-kappaB mediates PGE2 release via the expression of cyclooxygenase-2 and microsomal prostaglandin E synthase. FEBS Lett 547, 75-79.

6. Masuko-Hongo K, Berenbaum F, Humbert L, et al. (2004) Up-regulation of microsomal prostaglandin $\mathrm{E}$ synthase 1 in osteoarthritic human cartilage: critical roles of the ERK-1/2 and p38 signaling pathways. Arthritis Rheum 50, 2829-2838.

7. Russel MG, Engels LG, Muris JW, et al. (1998) Modern life's in the epidemiology of inflammatory bowel disease: a case-control study with special emphasis on nutritional factors. Eur J Gastroenterol Hepatol 10, 243-249.

8. Lansky EP \& Newman RA (2007) Punica granatum (pomegranate) and its potential for prevention and treatment of inflammation and cancer. J Ethnopharmacol 109, 177-206.

9. Larrosa M, Tomás-Barberán FA \& Espín JC (2006) The dietary hydrolysable tannin punicalagin releases ellagic acid that induces apoptosis in human colon adenocarcinoma Caco-2 cells by using the mitochondrial pathway. J Nutr Biochem 17, $611-625$.

10. Shukla M, Gupta K, Rasheed Z, et al. (2008) Consumption of hydrolyzable tannins-rich pomegranate extract suppresses inflammation and joint damage in rheumatoid arthritis. Nutrition 24, 733-743.

11. Shukla M, Gupta K, Rasheed Z, et al. (2008) Bioavailable constituents/metabolites of pomegranate (Punica granatum L.) preferentially inhibit COX-2 activity ex vivo and IL-1betainduced $\mathrm{PGE}_{2}$ production in human chondrocytes in vitro. J Inflamm (Lond) 13, 5-9.

12. Papoutsi Z, Kassi E, Chinou I, et al. (2008) Walnut extract (Juglans regia L.) and its component ellagic acid exhibit antiinflammatory activity in human aorta endothelial cells and osteoblastic activity in the cell line KS483. Br J Nutr 99, $715-722$.

13. Cerdá B, Cerón JJ, Tomás-Barberán FA, et al. (2003) Repeated oral administration of high doses of the pomegranate ellagitannin punicalagin to rats for 37 days is not toxic. J Agric Food Chem 51, 3493-3501.

14. Cerdá B, Espín JC, Parra S, et al. (2004) The potent in vitro antioxidant ellagitannins from pomegranate juice are metabolised into bioavailable but poor antioxidant hydroxy- $6 \mathrm{H}-$ dibenzopyran-6-one derivatives by the colonic microbiota of healthy humans. Eur J Nutr 43, 205-220.

15. Cerdá B, Periago P, Espín JC, et al. (2005) Identification of urolithin A as a metabolite produced by human colon microflora from ellagic acid and related compounds. J Agric Food Chem 53, 5571-5576.

16. González-Sarrías A, Azorín-Ortuño M, Yáñez-Gascón MJ, et al. (2009) Dissimilar in vitro and in vivo effects of ellagic acid and its microbiota-derived metabolites, urolithins, on the cytochrome P450 1A1. J Agric Food Chem 57, 5623-5632.

17. Larrosa M, González-Sarrías A, Yañéz-Gascón MJ, et al. (2010) Anti-inflammatory properties of a pomegranate extract and its metabolite urolithin-A in a colitis rat model and the effect of colon inflammation on the phenolic metabolism. J Nutr Biochem (In the Press).

18. Morgan DM (1998) Tetrazolium (MTT) assay for cellular viability and activity. Methods Mol Biol 79, 179-183.

19. Larrosa M, Luceri C, Vivoli E, et al. (2009) Polyphenol metabolites from colonic microbiota exert anti-inflammatory activity on experimental inflammation models. Mol Nutr Food Res 53, 1044-1054.

20. Jang M, Cai L, Udeani GO, et al. (1997) Cancer chemopreventive activity of resveratrol, a natural product derived from grapes. Science 275, 218-220.

21. Subbaramaiah K, Chung WJ, Michaluart P, et al. (1998) Resveratrol inhibits cyclooxygenase-2 transcription and activity in phorbol ester-treated human mammary epithelial cells. $J$ Biol Chem 273, 21875-21882.

22. Oh SH \& Lee BH (2003) Induction of apoptosis in human hepatoblastoma cells by tetrandrine via caspase-dependent Bid cleavage and cytochrome $c$ release. Biochem Pharmacol 66, $725-731$

23. Larrosa M, González-Sarrías A, García-Conesa MT, et al. (2006) Urolithins, ellagic acid-derived metabolites produced by human colonic microflora, exhibit estrogenic and antiestrogenic activities. J Agric Food Chem 54, 1611-1620.

24. Cerdá B, Tomás-Barberán FA \& Espín JC (2005) Metabolism of antioxidant and chemopreventive ellagitannins from strawberries, raspberries, walnuts, and oak-aged wine in humans: identification of biomarkers and individual variability. J Agric Food Chem 53, 227-235.

25. Faria A, Monteiro R, Mateus N, et al. (2007) Effect of pomegranate (Punica granatum) juice intake on hepatic oxidative stress. Eur J Nutr 46, 271-278.

26. Adams LS, Seeram NP, Aggarwal BB, et al. (2006) Pomegranate juice, total pomegranate ellagitannins, and punicalagin suppress inflammatory cell signalling in colon cancer cells. J Agric Food Chem 54, 980-985.

27. Cerdá B, Soto C, Albaladejo MD, et al. (2006) Pomegranate juice supplementation in chronic obstructive pulmonary disease: a 5-week randomized, double-blind, placebo-controlled trial. Eur J Clin Nutr 60, 245-253.

28. Espín JC, González-Barrio R, Cerdá B, et al. (2007) Iberian pig as a model to clarify obscure points in the bioavailability and metabolism of ellagitannins in humans. J Agric Food Chem 55, 10476-10485.

29. Mahida YR, Beltinger J, Makh S, et al. (1997) Adult human colonic subepithelial myofibroblasts express extracellular matrix proteins and cyclooxygenase-1 and -2. Am J Physiol 273, G1341-G1348.

30. Mifflin RC, Saada JI, Di Mari JF, et al. (2002) Regulation of COX-2 expression in human intestinal myofibroblasts: mechanisms of IL-1-mediated induction. Am J Physiol Cell Physiol 282, C824-C834.

31. Zhang Y, DeWitt DL, Murugesan S, et al. (2004) Novel lipidperoxidation- and cyclooxygenase-inhibitory tannins from Picrorhiza kurroa seeds. Chem Biodivers 1, 426-441.

32. Sadhu SK, Okuyama E, Fujimoto H, et al. (2006) Prostaglandin inhibitory and antioxidant components of Cistus laurifolius, a Turkish medicinal plant. J Ethnopharmacol 108, 371-378.

33. Chell S, Kaidi A, Williams AC, et al. (2006) Mediators of $\mathrm{PGE}_{2}$ synthesis and signalling downstream of COX-2 represent potential targets for the prevention/treatment of colorectal cancer. Biochim Biophys Acta 1766, 104-119.

34. Afaq F, Malik A, Syed D, et al. (2005) Pomegranate fruit extract modulates UV-B-mediated phosphorylation of mitogen-activated protein kinases and activation of nuclear factor kappa B in normal human epidermal keratinocytes paragraph sign. Photochem Photobiol 81, 38-45.

35. Newton R, Kuitert LM, Bergmann M, et al. (1997) Evidence for involvement of NF-kappaB in the transcriptional control of COX-2 gene expression by IL-1beta. Biochem Biophys Res Commun 237, 28-32.

36. Guan Z, Buckman SY, Miller BW, et al. (1998) Interleukin1beta-induced cyclooxygenase- 2 expression requires activation of both c-Jun $\mathrm{NH}_{2}$-terminal kinase and p38 MAPK signal 
pathways in rat renal mesangial cells. $J$ Biol Chem 273, 28670-28676.

37. LaPointe MC \& Isenović E (1999) Interleukin-1 $\beta$ regulation of inducible nitric oxide synthase and cyclooxygenase- 2 involves the p42/44 and p38 MAPK signaling pathways in cardiac myocytes. Hypertension 33, 276-282.

38. Yang CM, Chien CS, Hsiao LD, et al. (2002) Interleukin1 beta-induced cyclooxygenase- 2 expression is mediated through activation of p42/44 and p38 MAPKS, and NF-kappaB pathways in canine tracheal smooth muscle cells. Cell Signal 14, 899-911.

39. González-Sarrías A, Espín JC, Tomás-Barberán FA, et al (2009) Gene expression, cell cycle arrest and MAPK signalling regulation in Caco-2 cells exposed to ellagic acid and its metabolites, urolithins. Mol Nutr Food Res 53, 686-698.

40. Masamune A, Satoh M, Kikuta K, et al. (2005) Ellagic acid blocks activation of pancreatic stellate cells. Biochem Pharmacol 70, 869-878.

41. Krysan K, Reckamp KL, Dalwadi H, et al. (2005) Prostaglandin $\mathrm{E}_{2}$ activates mitogen-activated protein kinase/Erk pathway signaling and cell proliferation in non-small cell lung cancer cells in an epidermal growth factor receptor-independent manner. Cancer Res 65, 6275-6281.
42. Sirois J \& Richards JS (1993) Transcriptional regulation of the rat prostaglandin endoperoxide synthase 2 gene in granulosa cells. Evidence for the role of a cis-acting C/EBP beta promoter element. J Biol Chem 268, 21931-21938.

43. Inoue H, Yokoyama C, Hara S, et al. (1995) Transcriptional regulation of human prostaglandin-endoperoxide synthase-2 gene by lipopolysaccharide and phorbol ester in vascular endothelial cells. Involvement of both nuclear factor for interleukin-6 expression site and cAMP response element. J Biol Chem 270, 24965-24971.

44. Subbaramaiah K, Yoshimatsu K, Scherl E, et al. (2004) Microsomal prostaglandin E synthase-1 is overexpressed in inflammatory bowel disease. Evidence for involvement of the transcription factor Egr-1. J Biol Chem 279, 12647-12658.

45. Pascual RM, Carr EM, Seeds MC, et al. (2006) Regulatory features of interleukin-1beta-mediated prostaglandin $\mathrm{E}_{2}$ synthesis in airway smooth muscle. Am J Physiol Lung Cell Mol Physiol 290, L501-L508.

46. Meadows AL, Kong B, Berdichevsky M, et al. (2008) Metabolic and morphological differences between rapidly proliferating cancerous and normal breast epithelial cells. Biotechnol Prog 24, 334-341. 